\title{
BACs as Tools for the Study of Genomic Imprinting
}

\author{
S. J. Tunster, M. Van De Pette, and R. M. John \\ Cardiff School of Biosciences, Cardiff University, Cardiff CF10 3AX, UK \\ Correspondence should be addressed to R. M. John, johnrm@cf.ac.uk
}

Received 20 May 2010; Revised 20 July 2010; Accepted 19 October 2010

Academic Editor: Noelle E. Cockett

Copyright () 2011 S. J. Tunster et al. This is an open access article distributed under the Creative Commons Attribution License, which permits unrestricted use, distribution, and reproduction in any medium, provided the original work is properly cited.

\begin{abstract}
Genomic imprinting in mammals results in the expression of genes from only one parental allele. Imprinting occurs as a consequence of epigenetic marks set down either in the father's or the mother's germ line and affects a very specific category of mammalian gene. A greater understanding of this distinctive phenomenon can be gained from studies using large genomic clones, called bacterial artificial chromosomes (BACs). Here, we review the important applications of BACs to imprinting research, covering physical mapping studies and the use of BACs as transgenes in mice to study gene expression patterns, to identify imprinting centres, and to isolate the consequences of altered gene dosage. We also highlight the significant and unique advantages that rapid BAC engineering brings to genomic imprinting research.
\end{abstract}

\section{Introduction}

Genomic imprinting describes a unique class of genes that are expressed from only one parental allele as a consequence of epigenetic marks set down either in the father's or the mother's germ line [12] (Figure 1). Essentially, although two gene copies are physically present within each diploid somatic cell, only one gene copy is transcriptionally active, producing an RNA product. The first evidence that individual genes were imprinted came from studies on the mouse Insulin-like growth factor 2 (Igf2) gene [13]. An embryonic growth restriction phenotype was apparent in heterozygous offspring after paternal transmission of a targeted deletion of this locus initially suggesting haploinsufficiently. However, heterozygous animals also had unexpectedly low levels of expression of $I g f 2$ rather than the anticipated $50 \%$ reduction. Imprinting of the locus was subsequently demonstrated genetically [14]. In quick succession, a receptor for Igf2, Igf2r, and one of the most abundant RNAs in the developing mouse embryo, H19, were found to be maternally expressed [15, 16]. Thus, in short succession, allele-specific gene expression was demonstrated for three genes in mice. We now know of at least 80 protein-coding genes that are imprinted in both mouse and human. Many of these genes play important roles in early development, and many are physically linked within domains of both maternally- and paternally-expressed genes.
This work is summarized at http://www.mousebook.org/. Imprinted genes within domains are regulated by discrete genomic regions called imprinting centres (ICs) [17]. These regions, which can also be referred to as imprint control elements (ICEs) or imprint control regions (ICRs), are functionally defined by engineering-targeted deletions in mice [18-32]. Inheritance of an IC deletion through one parental germ line releases all the genes within the domain from their imprinted expression (loss of imprinting, LOI) whereas inheritance through the other parent's germ line generally, but not always, has no consequence. These ICs carry a DNA methylation imprint on one parental allele only, and studies on the DNA methyl-transferases (Dnmts) Dnmt3a and Dnmt3b and the accessory protein Dnmt3L demonstrate the necessity of de novo DNA methylation for the establishment of allele-specific gene expression [33-37].

Much of our understanding of the mechanism and function of genomic imprinting is based on data from the targeted deletion of imprinted genes, trans-factors or ICs in mice. However, while studies on loss of function are important for our understanding of gene function per se, imprinting is a dosage-related phenomenon. Altering the dosage of the imprinted gene is more informative with respect to the function of the imprint. Furthermore, while targeted deletions of ICs may confirm the requirement of a region for imprinting, further painstaking work is required 
TABLE 1: Summary of BAC transgene models used to test for autonomous imprinting.

\begin{tabular}{|c|c|c|c|c|}
\hline Gene (s) & Size & $\begin{array}{l}\text { Imprinting (Lines } \\
\text { imprinting/lines } \\
\text { tested) }\end{array}$ & Expression in embryo & Reference \\
\hline Nnat & $270 \mathrm{~kb} \mathrm{BAC}$ & $1 / 1$ & Full & {$[1]$} \\
\hline Nnat & $95 \mathrm{~kb} \mathrm{BAC}$ & $2 / 3$ & $\begin{array}{c}\text { Partial (lacking neural } \\
\text { enhancers) }\end{array}$ & {$[1]$} \\
\hline$C d k n 1 c$ & $260 \mathrm{~kb} \mathrm{BAC}$ & $0 / 2$ & Full $(C d k n 1 c)$ & {$[2]$} \\
\hline$C d k n 1 c$ & $85 \mathrm{~kb}$ BAC & $0 / 4$ & Partial & {$[2]$} \\
\hline Peg3/Zim1 & $120 \mathrm{~kb} \mathrm{BAC}$ & $1 / 3$ (Peg3 only) & $\begin{array}{l}\text { Neural expression of } \\
P e g 3 \text { but fails to } \\
\text { rescue Peg3-deficiency }\end{array}$ & {$[3]$} \\
\hline Gtl2 & $178 \mathrm{~kb} \mathrm{BAC}$ & $2 / 2$ & Partial & {$[4]$} \\
\hline ZAC1/HYMAI & $175 \mathrm{~kb}$ PAC & $2 / 2$ & $\begin{array}{l}\text { Full expression by in } \\
\text { situ }\end{array}$ & {$[5]$} \\
\hline Necdin and Magel & $109 \mathrm{~kb}^{*} \mathrm{BAC}$ & $0 / 1$ & Partial (Necdin) & {$[6]$} \\
\hline $\operatorname{Ig} f 2 r$ & $170 \mathrm{~kb} \mathrm{BAC}$ & $4 / 4$ & Full & [7] \\
\hline Dlk1/Gtl2 & $70 \mathrm{~kb}$ BAC & $0 / 3$ & Full (Dlk1) & {$[8]$} \\
\hline
\end{tabular}

TABLE 2: Functional consequence in transgenic overexpression models.

\begin{tabular}{|c|c|c|c|c|}
\hline Gene dosage & $\begin{array}{l}\text { Expressed } \\
\text { allele }\end{array}$ & Embryonic phenotype & EE phenotype & Reference \\
\hline $\begin{array}{l}2 \mathrm{X} C d k n 1 c \\
(1 \text { copy BAC) }\end{array}$ & Maternal & $\begin{array}{l}\text { E13.5 embryos } 80 \% \text { WT } \\
\text { Proportional decrease in the } \\
\text { weight of internal organs such as } \\
\text { kidney, lung, and liver }\end{array}$ & No placental expression & {$[9]$} \\
\hline $\begin{array}{l}3 \text { X Phlda2 and Slc22a18 } \\
(2 \text { copy BAC) }\end{array}$ & Maternal & $\begin{array}{l}\text { E13.5 embryos similar to WT } \\
\text { E16.5 embryos } 90 \% \text { WT weight } \\
\text { E18.5 embryos } 87 \% \text { WT }\end{array}$ & E14.5 placentae $80 \% \mathrm{WT}$ & {$[10,11]$} \\
\hline $\begin{array}{l}2 \mathrm{X} \text { Dlk1/Gtl2 } \\
\text { (4-7 copies BAC) }\end{array}$ & Paternal & E16.5 embryos 110\% WT & No placental expression & {$[8]$} \\
\hline
\end{tabular}

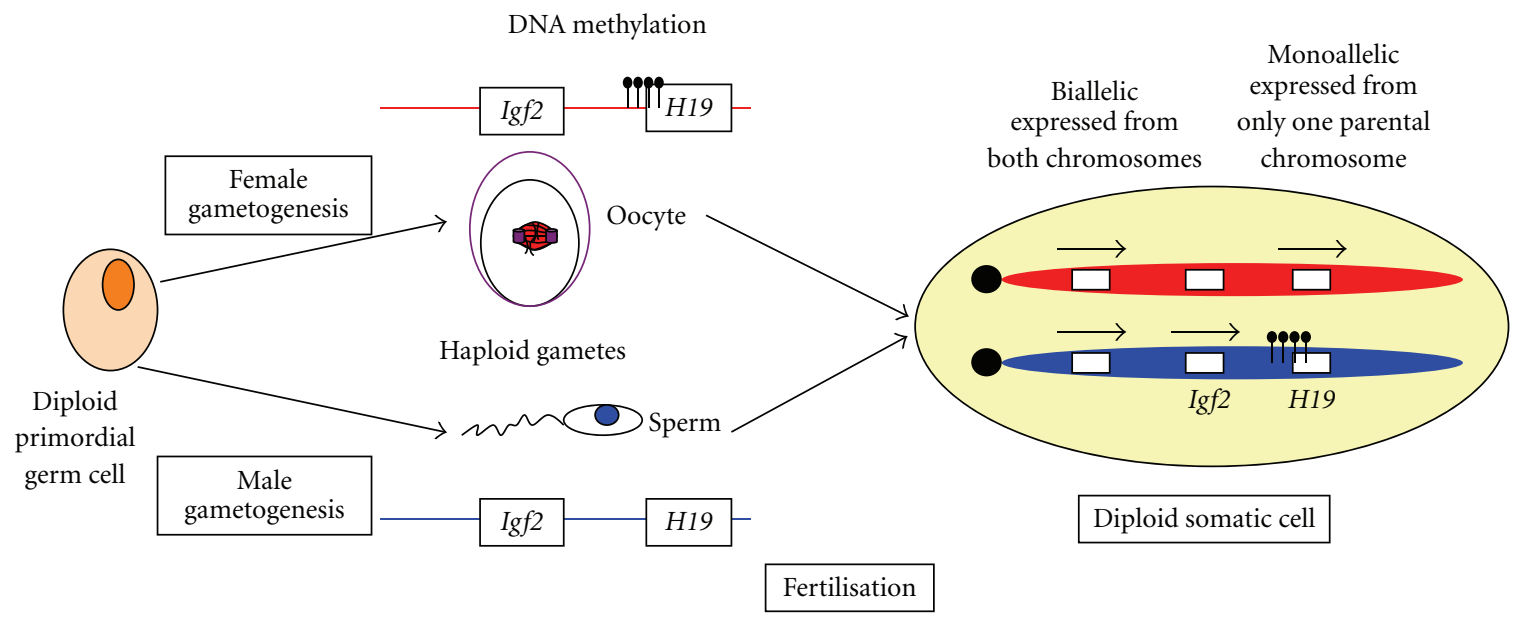

FIGURE 1: During mammalian gametogenesis, the diploid primordial germ cell undergoes meiosis to produce haploid male and female gametes. As meiosis progresses, specific DNA sequences acquire a DNA methylation imprint (black lollypops) in one parental germline but not the other. After fertilization, this imprint is recognized within the somatic cells establishing an imprinted domain. Maternal inheritance is indicated in red and paternal inheritance is indicated in blue. 


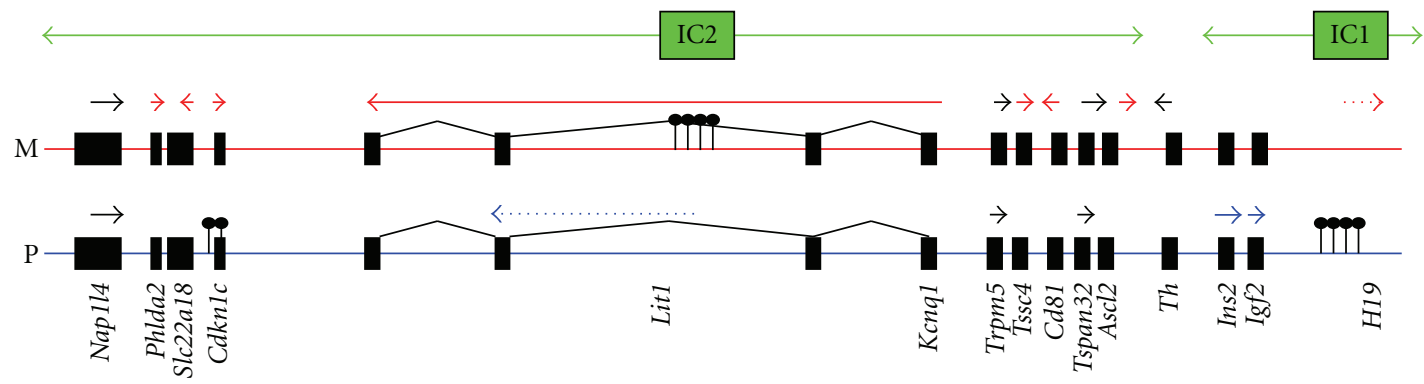

(a)

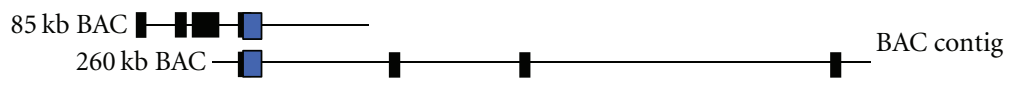

(b)

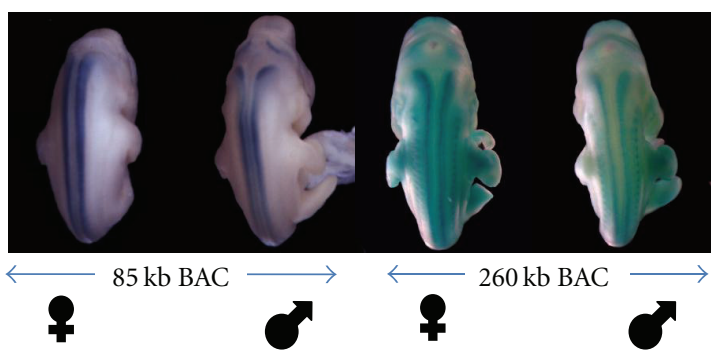

(c)

Figure 2: Mouse distal chromosome 7 contains two complex imprinted loci. (a) A diagrammatic representation of mouse distal chromosome 7 with maternal inheritance indicated in red and paternal inheritance indicated in blue. Genes are represented by the black boxes with the parent-of-origin expression indicated by either a red (maternal) or blue (paternal) arrow. Biallelic expression is indicated by a black arrow. Noncoding RNAs are represented by dashed arrows. DNA methylation is represented by the black lollypops. Green boxes indicate position of ICs whilst green doubleheaded arrows indicate the region influenced by the IC. (b) BAC clones that are used to examine expression, imprinting, and function. Blue boxes indicate the position of lacZ insertion. (c) Images of transgenic embryos inheriting the modified BACs through either the maternal or paternal germline as indicated by gender symbols below image. Expression of $C d k n 1 c$ from the BAC is revealed by lacZ reporter expression.

to dissect the function of these regions and to identify each element of the imprinting process. Transgenes provide an essential tool to our armory. A transgene-based approach can provide information both on the mechanism of imprinting and also the functional consequences of increased gene expression in a single model. In this respect, transgenes based on bacterial artificial chromosomes (BACs) have been of particular value.

\section{BACs}

BACs were first developed as a large insert clone system to facilitate the construction of an orderly set of overlapping clones as tools for the Human Genome Project [38]. BACs are single copy replicons based on the naturally occurring Escherichia coli fertility plasmid. This vector system is capable of cloning and propagating large DNA fragments with an average insert size of $150 \mathrm{~kb}$ and a maximum insert size of $700 \mathrm{~kb}$. The key advantage of BACs over other large insert technologies is their stability in culture and ease of manipulation. These qualities initially rendered them an ideal resource for physically mapping genomes and they have been used in almost all the genome sequencing projects [38]. One major advantage that large insert clones bring to transgenic research is that they are more likely to contain the necessary promoter, enhancer, and silencer combination to mimic the natural expression of the gene of interest. The advantages of the BAC transgenic approach compared to a conventional transgenic approach have been discussed extensively elsewhere [39]. However, there are several advantages that BACs bring that are specific to imprinting research. Firstly, the imprinting capacity of BACs carrying both target genes and putative ICs can be examined outside the normal chromosomal context. Secondly, BACs can be used to study the developmental consequence of accurate but excess expression of single genes. And thirdly, their amenability to modification techniques to insert or delete sequences and to alter sequences as discrete as a single point mutation [40-44] makes them a powerful tool for addressing both mechanistic and functional questions. BACs can be modified rapidly in vitro, with an average construction time of less than 4 weeks. Once the modified $\mathrm{BAC}$ is made, transgenic founders can be generated by the pronuclear injection of the construct into fertilised eggs to generate transgenic founders. Thus, modified BACs can provide an important additional tool, alongside traditional targeting of endogenous loci in embryonic stem (ES) cells. 


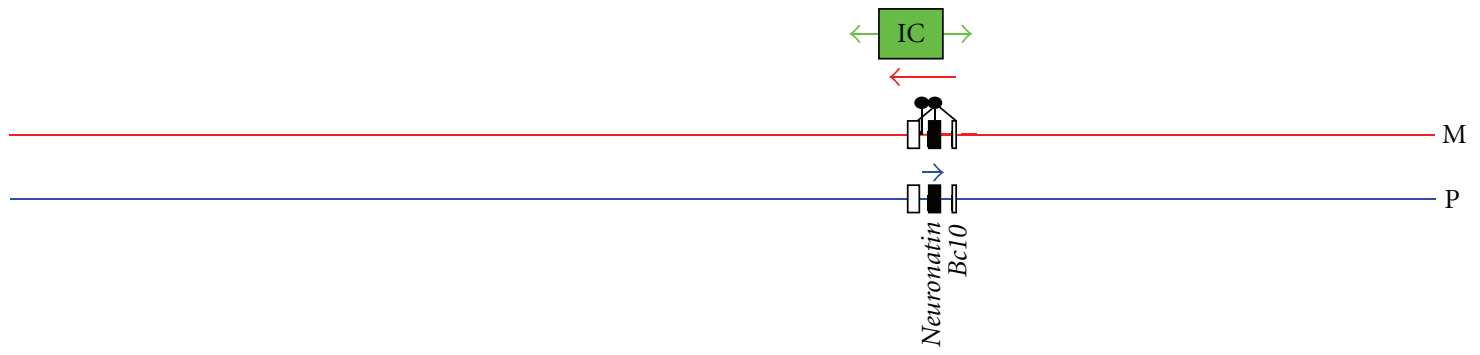

(a)

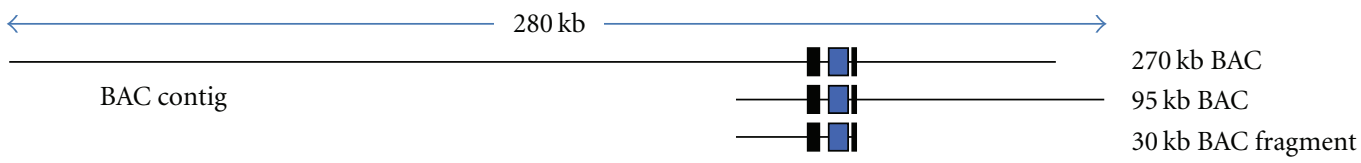

(b)

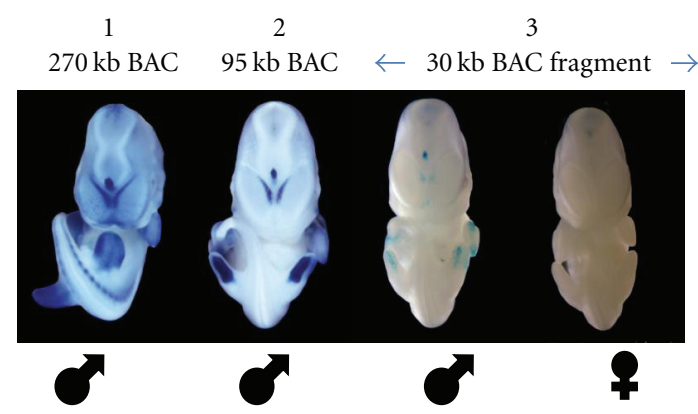

(c)

FIGURE 3: Mouse distal chromosome 2 contains a minimalistic imprinted locus. (a) A diagrammatic representation of mouse distal chromosome 2 imprinted region with maternal inheritance indicated in red and paternal inheritance indicated in blue. Genes are represented by the boxes with the parent-of-origin expression indicated by either a red (maternal) or blue (paternal) arrow. DNA methylation is represented by the black lollypops. Green box indicates position of proposed IC whilst green doubleheaded arrow indicates the known region influenced by this IC. (b) BAC clones and subclone used to examine expression, imprinting, and function. Blue boxes indicate the position of lacZ reporter. (c) Images of transgenic embryos inheriting the modified BACs through either the maternal or paternal germline as indicated by gender symbols below image. Expression of Neuronatin from the transgenic insertion is revealed by lacZ reporter expression.

\section{Physical Maps of Imprinted Domains}

BACs were first applied to imprinting research in order to generate physical maps of imprinted domains. Early work suggested that imprinted genes were not randomly scattered throughout the chromosomes but localized to discrete domains containing both maternally- and paternallyexpressed genes. This featuristic organization is ideally suited to the construction of contigs of large genomic clones. Firstly, to provide detailed information about the physical organization of the known genes within the domain. Secondly, to extend the contigs to identify physically linked genes and test their imprint status. Thirdly, and perhaps most importantly, to identify nongenic regions of conservation between different species as likely ICs.

The first imprinted region to be physically mapped using BACs was human chromosome 11p15.5 [45]. This region has been the focus of intense study because of the association with the classic imprinting disorder, Beckwith Wiedemann Syndrome (OMIM 130650; BWS). First human and then mouse contigs [2, 46-48] provided templates for sequencing to reveal information on the location of genes, their physical structure, and also the location of genomic sequence features conserved between mouse and human. This approach was important in identifying both a conserved putative IC (IC2) carrying a germ line imprint and also novel genes located inside and outside the previously proposed boundaries of the imprinting cluster $[49,50]$. BACs have subsequently been similarly employed to physically characterize additional imprinted loci including SNRPN, MEST/PEG1, Peg3/Zim1, Dlk2/Gtl2, Gnas, and Neuronatin [1, 3, 51-56].

\section{BACs and Studies on the Evolution of Imprinting}

The construction of BAC libraries for a variety of species provides unprecedented resources for advancing the ongoing research into genomic imprinting. These resources have particular relevance to studies on imprinting because, while imprinting has been demonstrated in marsupial and eutherian mammals, it has not been reported in monotremes (platypus and echidna) or nonmammalian vertebrates. This suggests that imprinting arose sometime after the divergence 
of monotremes (prototherians) from therian mammals, which has important implications for understanding the rational for this phenomenon [57]. Comparing genomic regions in key representatives of mammalian diversity and phylogeny will be of great value in unlocking further secrets of genomic imprinting.

As the most intensively investigated imprinted domain in the human and mouse genomes, the BWS imprinted region has also been scrutinized in nonmammalian vertebrates through the isolation of BAC clones $[58,59]$. This work was important in establishing that the imprinted genes within this domain were physically linked prior to acquisition of their imprint. Orthologues of a number of imprinted genes have also been isolated from BAC libraries constructed from the genomes of the tammar wallaby (marsupial) and the platypus (monotreme) [60]. Mapping these BACs to their respective chromosomes demonstrated that these imprinted gene orthologues existed on separate chromosomes prior to the evolution of imprinting. This finding suggests that genomic imprinting evolved independently from X-inactivation, despite similarities in the epigenetic mechanisms directing these two processes.

In eutherian mammals, imprinted domains are regulated by imprinting centres [17]. Using these DNA sequences directly as probes to identify orthologous regions in other species has not been successful. This is most likely due to their high GC content (sticky probes) and the relatively low conservation of DNA sequence, even between human and mouse. However, BACs can be identified that span regions predicted to contain ICs by using sequences from nearby protein-coding genes as baits. As an example, an SGCE/PEG10 tammar wallaby BAC was isolated using a mouse Sgce cDNA as a probe [61]. The BAC also contained a differentially methylated region (DMR) equivalent to that observed in eutherians providing the first evidence that DNA methylation is a conserved feature of the imprinting mechanism. This work highlighted another added value of BACs in cross-species studies. The tammar wallaby PEG10 gene was not identified in a direct screen but by primer walking from the physically-linked SGCE. Employing the same methodology, researchers were unable to identify any sequence with similarity to PEG10 within the platypus $S G C E$ BAC contig, thus, demonstrating that the PEG10 was inserted into the genome after monotremes diverged from the other lineages. Thus, not only has the mechanism for imprinting genes arisen at a critical time in the evolution of modern mammals, but also new genes have been added to the genome with entirely novel functions.

\section{BACs as Transgenes}

In addition to physical mapping studies, BACs have provided useful tools for establishing the expression and imprinting capabilities of specific genomic regions within imprinted loci (Table 1). Putative ICs for imprinted domains can initially be identified by their epigenetic characteristics (differential DNA methylation). These regions can subsequently be functionally defined by targeted deletion of the endogenous locus (see earlier). Another stringent test for imprint control regions is to examine their function at ectopic loci. If a sequence can direct imprinting when integrated randomly into the genome, this suggests that all the component parts of the imprinting mechanism are contained within this sequence.

The first transgenic studies of this sort relied on plasmidbased transgenes. However, the smaller transgenes were of limited use primarily because the site of integration can influence both expression and imprinting [62] but also because small transgenes were less likely to carry all the required elements to recapitulate expression of the endogenous locus. These disadvantages were overcome by increasing the size of the genomic region included in the transgene. The Igf2/H19 locus on mouse distal chromosome 7 and the Igf2r locus on mouse chromosome 17 were the first imprinted regions to be transgenically dissected using large genomic clones $[63,64]$. YACs were used in both cases. Imprinted expression was reliably established away from the respective domains indicating that the cis-sequences required to establish imprinting lay within the genomic regions encompassed by these YACs. BACs have largely rendered YACs obsolete for these types of study because of their relative stability in culture and ease of DNA preparation [65] and, most importantly, the development of technologies to insert or remove specific sequences, reviewed recently $[39,66]$. Sequences, such as reporters, can be homologously recombined into BACs to provide useful information on both spatial and imprinted expression. Where BACs contain more than one gene or where overexpression might be nonviable, homologous recombination can be used to inactivate gene loci $[1,2,9,10]$. As a future goal, putative ICs could be mutated at the single nucleotide level to explore mechanistic questions or conditionally targeted in order to examine the temporal requirement for these sequences. Although these latter procedures can be performed at the endogenous IC, the major advantage that BAC modification protocols have over targeted homologous recombination in ES cells is speed. Once a targeting vector is constructed, BACs can be modified in a matter of weeks and then injected into fertilized mouse oocytes to generate founders within a few months.

Cdkn1c (previously known as $p 57^{\text {Kip2 }}$ ) and Neuronatin (previously known as Peg5) were the first imprinted loci to be mechanistically explored using BACs $[1,2]$. Cdknlc maps to the BWS imprinted region on mouse distal chromosome $7 /$ Human chromosome $11 \mathrm{p} 15$. This is one of the most complex imprinted regions in mice containing at least 18 maternally- and paternally-expressed genes and three DMRs (Figure 2(a)). In mice, the region can be separated mechanistically into two distinct domains, termed the IC1 and IC2 domains [20-22, 26, 67]. Cdkn1c maps within the IC2 region. The fact that the regulatory elements were located at a distance from $C d k n 1 c$ was first suggested by studies on a $38 \mathrm{~kb}$ cosmid-based transgene spanning the human CDKN1C locus [68]. Despite containing $20 \mathrm{~kb}$ of sequence upstream and $15 \mathrm{~kb}$ of sequence downstream of the gene, CDKN1C was not expressed from the human transgene in multiple lines. Transgenic expression of $C d k n 1 c$ was only achieved using larger murine BAC-based transgenes suggesting the existence of distantly-located enhancers 
[2]. Two BACs spanning the murine Cdkn1c gene, of 85 and $260 \mathrm{~kb}$, respectively, were engineered to include a $\beta$ galactosidase reporter under the control of the Cdkn1c promoter (Figure 2(b)). Whole-mount LacZ analyses provided easy access to the expression pattern of the $C d k n 1 c$ gene under control of regulatory elements within the BAC. From the smaller BAC, Cdkn1c-lacZ was expressed in a subset of tissues in which the endogenous $C d k n 1 c$ locus was expressed whereas the much larger $260 \mathrm{~kb}$ BAC drove expression in all embryonic tissues (Figure 2(c)). Enhancers for extraembryonic tissues lay outside the $320 \mathrm{~kb}$ region scanned. The murine $C d k n 1 c$ gene is spanned by a DMR [68], but neither BAC transgene autonomously imprinted Cdkn1c [2]. A second DMR within the IC2 region, called KvDMR1, has now been functionally defined as the imprinting centre for the region controlling imprinted expression of $C d k n 1 c$ and the other maternally-expressed genes [26]. This imprinting centre is contained within the $260 \mathrm{~kb} \mathrm{Cdknlc} \mathrm{BAC} \mathrm{so,} \mathrm{in}$ theory, it should imprint at ectopic chromosomal loci. This was not the case suggesting that KvDMR1 requires additional elements to function as an IC. Currently, only an $800 \mathrm{~kb}$ YAC, which encompasses almost the entire IC2 domain, has the capacity to imprint $C d k n 1 c$ [67].

Adjacent to the IC2 domain lies the IC1 domain, which spans Igf2, Ins2, and H19 (Figure 2(a)). Igf2/H19 were initially shown to imprint at ectopic loci from a $130 \mathrm{~kb}$ YAC [63], and a $137 \mathrm{~kb}$ BAC was used to further refine the minimal region required to imprint $H 19$ to $-7 \mathrm{~kb}$ and $+35 \mathrm{~kb}$ of the $H 19$ promoter [69]. This region contains the functionally defined IC just upstream of H19 [20-22]. Although smaller transgenes can drive imprinted expression of H19, they do so unreliably and without inducing germline DNA methylation at the IC whereas the IC within the larger BAC clone does become DNA methylated in the male germline [70]. These data suggest that, while ICs initiate the imprinting mechanism, the surrounding sequence is important in interpreting and maintaining the process.

The paternally expressed Neuronatin gene maps to one of the least complex imprinted domains, located on mouse distal chromosome 2/Human chromosome 20 (Figure 3(a)). Neuronatin is not located within a cluster of imprinted genes but lies within the intron of a second gene, Blcap (previously known as Bc10) [1]. Blcap shows a maternalallele bias in expression in tissues where Neuronatin is highly expressed, transcriptional interference rather than a direct imprint [71]. The body of the Neuronatin gene carries direct differential DNA methylation on the maternal allele and within this DMR, there is a smaller region that exhibits the biochemical characteristics of an IC [1, 72]. Transgenic mice engineered with a series of BAC clones modified to include a $\beta$-galactosidase reporter under the control of the Neuronatin promoter were used to demonstrate that the minimum sequence required to imprint Neuronatin was approximately $30 \mathrm{~kb}$ and, indeed, encompassed the putative IC (Figures 3(b) and $3(\mathrm{c})$ ). In addition, these studies revealed that enhancers for tissue-specific expression of Neuronatin were primarily located upstream of the putative IC and that some of them lay at a significant distance from the body of the gene.
Overlapping BAC transgenes have also been used to explore the Delta-like1 (Dlk1)/Gene-trap locus2 (Gtl2) imprinted domain on mouse chromosome 12. Imprinted expression of Gtl2 was reported from a $178 \mathrm{~kb}$ BAC that spans a region from $3.5 \mathrm{~kb}$ upstream of the physically linked Delta-like1 (Dlk1) gene to $69 \mathrm{~kb}$ downstream of Gtl2 [4]. This Dlk1/Gtl2 BAC drove expression of Gtl2 in a subset of tissues in which the endogenous locus is expressed, but $D l k 1$ was not expressed from this BAC in any tissue. Dlk1 was expressed from a smaller $70 \mathrm{~kb}$ BAC encompassing more sequence upstream of $D l k 1$ gene, but expression was not imprinted, thus confirming the location of the IC linked to Gtl2 [8].

Some BAC studies are more difficult to interpret. The $120 \mathrm{~kb}$ BAC spanning the Peg3/Zim1 locus, which contains $20 \mathrm{~kb}$ of sequence upstream of $P e g 3$ and $80 \mathrm{~kb}$ of sequence downstream, showed imprinted expression of Peg3 in one transgenic line but not in two others [3]. Peg3 is spanned by a germline DMR $[73,74]$ which suggests that the IC for Peg3 is contained within this $120 \mathrm{~kb}$ BAC. Like the ICs for H19/Igf2 and Cdkn1c, perhaps the Peg3 IC is reliant on additional sequences to fully communicate the imprinting signal.

\section{Functional Studies of Imprinted Genes}

Providing the appropriate regulatory elements that are also present, genes are expressed from BACs with spatial and temporal accuracy and at similar levels to the endogenous loci, predominantly without being affected by the site of integration [75]. Consequently, BACs can be used to precisely engineer increased dosage of gene loci. This has particular relevance to studies on imprinted loci because gene dosage is key to the phenomenon. Genomic imprinting alters the expression level of a particular gene from one parental allele without altering its essential function. Therefore, engineering altered dosage of an imprinted gene addresses the function of the imprint as well as the function of the gene. Modifying the endogenous locus by targeting an IC is one route to engineering biallelic expression, recently reviewed [76]. These models can provide excellent tools for understanding the consequences of increased gene dosage, particularly where the IC controls a few well-characterized targets. However, LOI can mean increased dosage of some genes (gene activation) and loss of expression of others (gene silencing). Furthermore, the majority of imprinted domains are complex and not fully characterized. Interpreting the results of these studies with respect to individual genes is not straightforward.

The critical advantage that BAC transgenes provide over LOI models for exploring the function of imprinting is that the exact nature and number of genes is precisely defined by the transgenic sequence assigning phenotypes unequivocally to the gene sequences within the transgene. BACs may also be useful in "rescuing" phenotypes associated with LOI of complex domains, particularly in cases where loss of expression of one gene within the domain preclude a phenotypic assessment of other genes. Most importantly, the ability to rapidly modify BACs is particularly helpful 
in situations where more than one gene is present in close proximity.

Functional studies performed on the imprinted locus containing the three closely linked genes, Phlda2, Slc22a18, and $C d k n 1 c$, provide a textbook example of the advantages that BACs bring to imprinting research [9-11]. These three genes are all contained within a $40 \mathrm{~kb}$ region of IC2 domain. Their close proximity means that it would likely be impossible to separate the genes on individual genomic fragments and still maintain appropriate temporal and spatial expression. However, when an $85 \mathrm{~kb}$ transgene spanning this locus was found to contain the placental enhancers for Phlda2 and Slc22a18 but not for Cdkn1c, this allowed the assignment of a placental stunting phenotype to overexpression of just Phlda2 and/or Slc22a18 [11]. The placental stunting phenotype is reciprocal to placentomegaly induced by loss of expression of Phlda2 [77]. This suggested that Phlda2 acts as a rheostat for placental growth, with overgrowth after gene deletion and growth retardation after loss of imprinting [11]. A key role for Phlda2 in regulating placental weight and glycogen storage was genetically verified by combining a single copy of the BAC transgene with a maternally-inherited targeted deletion of Phlda2 to rescue Phlda2 overexpression [10]. Essentially, these double transgenic mice have wildtype levels of Phlda2, but Slc22a18 remains in excess. Their placentae were phenotypically indistinguishable from wild type, thus excluding a role for Slc22a18 in placental growth restriction.

In addition to exhibiting placental growth restriction, the Cdkn1c/Phlda2/Slc22a18 BAC transgene also restricted embryonic growth from E13.5. This early growth restriction phenotype was genetically assigned to excess $C d k n 1 c$ and not excess Phlda2 or Slc22a18 by engineering a modification to abolish $C d k n 1 c$ expression from the BAC. Mice carrying the modified BAC, with excess Phlda2 and Slc22a18 expression but normal levels of $C d k n 1 c$, were not growth restricted at E13.5, thus providing genetic evidence that $C d k n 1 c$ encodes a potent negative regulator of embryonic growth [9]. In addition to assigning the early embryonic phenotype to excess $C d k n 1 c$, ablation of $C d k n 1 c$ function from the BAC transgene uncovered a second distinct growth restriction phenotype. Mice carrying the modified BAC (no Cdknlc overexpression) were the same weight as nontransgenic embryos at E13.5 but showed a progressive loss of growth potential later in gestation, being $13 \%$ lighter that controls by birth $[10,11]$. This suggests a role for Phlda2 and/or Slc22a18 in regulating late embryonic growth. This phenotype would have been missed by any other approach as the earlier $C d k n 1 c$-induced growth restriction phenotype effectively obscures the later phenotype. This study perfectly illustrates the way in which subtle phenotypes associated with altered expression of one imprinted gene within a domain can be masked by other closely linked genes.

Functional BAC-based studies have also been performed on the Dlk1 locus [8] (Table 2). Dlk1, also known as Preadipocyte factor 1 (Pref-1), encodes an inhibitor of adipocyte differentiation. Loss of expression of this paternally-expressed gene in mice results in growth retardation, obesity, abnormal eyelids, skeletal malformation, and increased serum lipid metabolites [78]. Examining the consequence of overexpression of $D l k 1$ in isolation was only achievable using a $70 \mathrm{~kb}$ BAC transgene containing $49.4 \mathrm{~kb}$ of sequence upstream of $D l \mathrm{kl}$ and $18 \mathrm{~kb}$ downstream of the Dlkl transcriptional start site. In contrast to the studies on Cdkn1c and Phlda2, Dlk1 was expressed at approximately the endogenous level from the BAC transgene regardless of copy number. However, a triple dose of Dlk1 was achieved by generating mice homozygous for the transgene. The $70 \mathrm{~kb}$ BAC recapitulated the spatiotemporal expression of the Dlk1 in embryonic tissues but not in the placenta. The study revealed an intriguing dual role for $D l k 1$ in driving embryonic overgrowth but with significantly reduced fitness after birth, demonstrating that Dlk1 is a dosage-critical gene within its domain, a key principle of imprinting.

\section{Future Work}

Initial studies on BACs have demonstrated their importance in both dissecting imprinting mechanisms and understanding imprinting function. Our ability to target BAC transgenes to specific loci as single copies would improve this technology. Such an approach would be useful in rigorously testing the imprinting capacity of different BAC clones within a single chromosomal location. Single-copy BACs would also "restore" biallelic expression to specific imprinted loci, a critical component of functional studies. Current approaches to generate BAC transgenic mice involve either pronuclear microinjection into fertilised eggs or electroporation into ES cells. Both these techniques result in the random integration of BAC clones into the mouse genome. This can cause variability due to differences in the copy number of the BAC and also position effects caused by the site of integration, albeit with low frequency compared to plasmid-based clones. As a result, multiple independent founder lines must be analysed. In addition, multiple copy number integrations can result in high levels of gene expression, which are less relevant to studies on genomic imprinting. Recently, BACs have been modified to contain the sequences necessary for homologous recombination into, and complementation of, the partially deleted hypoxanthine phosphoribosyltransferase (Hprt) locus in ES cells with positive selection for Hprt to achieve single-copy integrations [79]. Further developments could be based on recombining pre-existing loxP sites within some BAC vectors and one inserted at the Rosa26 locus [80], bypassing the necessity for any modification of the BAC.

BACs may be useful in addressing further important questions. For example, we know that ICs are required to establish imprinted expression within their domains. However, what happens if these ICs are deleted after the imprint has been established? Can domains maintain their imprinted status in the absence of continued signaling from their ICs? The ability to conditionally target ICs will provide important information on their role initiation of the imprint verses maintenance of imprinting. This is not a specific advantage to BACs since loxP sites can be targeted at the endogenous locus or to a BAC. However, performing these studies on a BAC clone would circumvent cases where loss 
of imprinting at the endogenous locus results in embryonic lethality, allowing studies in the adult.

Our ability to rapidly modify BACs in vitro would also make two-step sequential modifications more practical. BAC recombineering could then be used to generate a single clone containing two different regions flanked either by loxP-loxP sites or by FRT-FRT/loxP sites allowing sequential deletion of these regions in vivo Cre- and FLPe-recombinases, respectively.

In addition, modified BAC clones are now being used themselves as targeting vectors. Plasmid-based targeting vectors cover relatively short regions of the genome of a few kilobases. BACs can be used to generate targeting vectors where the two loxP sites are placed far apart. Such an approach would facilitate the generation of models aimed at the conditional deletion of larger genomic regions spanning two or more genes.

In summary, we have provided key examples of how BAC transgenesis has so far provided a powerful tool to study genomic imprinting. BACs can be used to address both mechanistic and functional questions. Our ability to rapidly modify BACs in vitro suggests that they have the potential to significantly further our understanding of genomic imprinting.

\section{Abbreviations}

IC: Imprinting centre

LOI: Loss of imprinting

DMR: Differentially methylated region

YAC: Yeast artificial chromosome

BAC: Bacterial artificial chromosome.

\section{Acknowledgments}

S. J. Tunster was supported by BBSRC Grant no. BB/ G015465, and M. Van De Pette holds a BBSRC Ph.D. studentship.

\section{References}

[1] R. M. John, S. A. J. R. Aparicio, J. F.-X. Ainscough et al., "Imprinted expression of Neuronatin from modified BAC transgenes reveals regulation by distinct and distant enhancers," Developmental Biology, vol. 236, no. 2, pp. 387399, 2001.

[2] R. M. John, J. F.-X. Ainscough, S. C. Barton, and M. Azim Surani, "Distant cis-elements regulate imprinted expression of the mouse p57Kip2 (Cdkn1c)gene: implications for the human disorder, Beckwith-Wiedemann syndrome," Human Molecular Genetics, vol. 10, no. 15, pp. 1601-1609, 2001.

[3] I. Y. Y. Szeto, S. C. Barton, E. B. Keverne, and M. A. Surani, "Analysis of imprinted murine Peg3 locus in transgenic mice," Mammalian Genome, vol. 15, no. 4, pp. 284-295, 2004.

[4] A. Yevtodiyenko, E. Y. Steshina, S. C. Farner, J. M. Levorse, and J. V. Schmidt, "A 178-kb BAC transgene imprints the mouse Gtl2 gene and localizes tissue-specific regulatory elements," Genomics, vol. 84, no. 2, pp. 277-287, 2004.

[5] D. Ma, J. P. M. Shield, W. Dean et al., "Impaired glucose homeostasis in transgenic mice expressing the human transient neonatal diabetes mellitus locus, TNDM," Journal of Clinical Investigation, vol. 114, no. 3, pp. 339-348, 2004.

[6] F. Watrin, E. Le Meur, N. Roeckel, M.-A. Ripoche, L. Dandolo, and F. Muscatelli, "The Prader-Willi syndrome murine imprinting center is not involved in the spatio-temporal transcriptional regulation of the Necdin gene," BMC Genetics, vol. 6, article no. 1, 2005.

[7] T. L. Wise and D. D. Pravtcheva, "Delayed onset of Igf2induced mammary tumors in Igf2r transgenic mice," Cancer Research, vol. 66, no. 3, pp. 1327-1336, 2006.

[8] S. T. Da Rocha, M. Charalambous, S.-P. Lin et al., "Gene dosage effects of the imprinted delta-like homologue 1 (Dlk1/Pref1) in development: implications for the evolution of imprinting," PLoS Genetics, vol. 5, no. 2, article no. e1000392, 2009.

[9] S. C. Andrews, M. D. Wood, S. J. Tunster, S. C. Barton, M. A. Surani, and R. M. John, "Cdkn1c (p57Kip2) is the major regulator of embryonic growth within its imprinted domain on mouse distal chromosome 7," BMC Developmental Biology, vol. 7, article no. 53, 2007.

[10] S. J. Tunster, B. Tycko, and R. M. John, “The imprinted Phlda2 gene regulates extraembryonic energy stores," Molecular and Cellular Biology, vol. 30, no. 1, pp. 295-306, 2010.

[11] M. Salas, R. John, A. Saxena et al., "Placental growth retardation due to loss of imprinting of Phlda2," Mechanisms of Development, vol. 121, no. 10, pp. 1199-1210, 2004.

[12] M. A. Surani, "Imprinting and the initiation of gene silencing in the germ line," Cell, vol. 93, no. 3, pp. 309-312, 1998.

[13] T. M. DeChiara, A. Efstratiadis, and E. J. Robertson, "A growth-deficiency phenotype in heterozygous mice carrying an insulin-like growth factor II gene disrupted by targeting," Nature, vol. 345, no. 6270, pp. 78-80, 1990.

[14] T. M. DeChiara, E. J. Robertson, and A. Efstratiadis, "Parental imprinting of the mouse insulin-like growth factor II gene," Cell, vol. 64, no. 4, pp. 849-859, 1991.

[15] D. P. Barlow, R. Stoger, B. G. Herrmann, K. Saito, and N. Schweifer, "The mouse insulin-like growth factor type-2 receptor is imprinted and closely linked to the Tme locus," Nature, vol. 349, no. 6304, pp. 84-87, 1991.

[16] M. S. Bartolomei, S. Zemel, and S. M. Tilghman, "Parental imprinting of the mouse H19 gene," Nature, vol. 351, no. 6322, pp. 153-155, 1991.

[17] R. M. John and M. A. Surani, "Imprinted genes and regulation of gene expression by epigenetic inheritance," Current Opinion in Cell Biology, vol. 8, no. 3, pp. 348-353, 1996.

[18] C. M. Williamson, S. T. Ball, W. T. Nottingham et al., "A cisacting control region is required exclusively for the tissuespecific imprinting of Gnas," Nature Genetics, vol. 36, no. 8, pp. 894-899, 2004.

[19] C. M. Williamson, M. D. Turner, S. T. Ball et al., "Identification of an imprinting control region affecting the expression of all transcripts in the Gnas cluster," Nature Genetics, vol. 38, no. 3, pp. 350-355, 2006.

[20] J. L. Thorvaldsen, K. L. Duran, and M. S. Bartolomei, "Deletion of the $H 19$ differentially methylated domain results in loss of imprinted expression of H19 and Igf2," Genes and Development, vol. 12, no. 23, pp. 3693-3702, 1998.

[21] M.-A. Ripoche, C. Kress, F. Poirier, and L. Dandolo, "Deletion of the H19 transcription unit reveals the existence of a putative imprinting control element," Genes and Development, vol. 11, no. 12, pp. 1596-1604, 1997.

[22] D. R. Esquiliano, W. Guo, L. Liang, P. Dikkes, and M. F. Lopez, "Placental glycogen stores are increased in mice with $H 19$ null 
mutations but not in those with insulin or IGF type 1 receptor mutations," Placenta, vol. 30, no. 8, pp. 693-699, 2009.

[23] S. J. Chamberlain and C. I. Brannan, "The Prader-Willi syndrome imprinting center activates the paternally expressed murine Ube3a antisense transcript but represses paternal Ube3a," Genomics, vol. 73, no. 3, pp. 316-322, 2001.

[24] T. Yang, T. E. Adamson, J. L. Resnick et al., "A mouse model for Prader-Willi syndrome imprinting-centre mutations," Nature Genetics, vol. 19, no. 1, pp. 25-31, 1998.

[25] M.-Y. Wu, K.-S. Chen, J. Bressler, A. Hou, T.-F. Tsai, and A. L. Beaudet, "Mouse imprinting defect mutations that model angelman syndrome," Genesis, vol. 44, no. 1, pp. 12-22, 2006.

[26] G. V. Fitzpatrick, P. D. Soloway, and M. J. Higgins, "Regional loss of imprinting and growth deficiency in mice with a targeted deletion of KvDMR1," Nature Genetics, vol. 32, no. 3, pp. 426-431, 2002.

[27] H. Shiura, N. Miyoshi, A. Konishi et al., "Meg1/Grb10 overexpression causes postnatal growth retardation and insulin resistance via negative modulation of the IGF1R and IR cascades," Biochemical and Biophysical Research Communications, vol. 329, no. 3, pp. 909-916, 2005.

[28] H. Shiura, K. Nakamura, T. Hikichi et al., "Paternal deletion of Meg1/Grb10 DMR causes maternalization of the Meg1/ Grb10 cluster in mouse proximal chromosome 11 leading to severe pre- and postnatal growth retardation," Human Molecular Genetics, vol. 18, no. 8, pp. 1424-1438, 2009.

[29] S.-P. Lin, P. Coan, S. T. da Rocha et al., "Differential regulation of imprinting in the murine embryo and placenta by the Dlk1Dio3 imprinting control region," Development, vol. 134, no. 2, pp. 417-426, 2007.

[30] S.-P. Lin, N. Youngson, S. Takada et al., "Asymmetric regulation of imprinting on the maternal and paternal chromosomes at the Dlk1-Gtl2 imprinted cluster on mouse chromosome 12," Nature Genetics, vol. 35, no. 1, pp. 97-102, 2003.

[31] A. Wutz, H. C. Theussl, J. Dausman, R. Jaenisch, D. P. Barlow, and E. F. Wagner, "Non-imprinted lgf2r expression decreases growth and rescues the Tme mutation in mice," Development, vol. 128, no. 10, pp. 1881-1887, 2001.

[32] B. Bielinska, S. M. Blaydes, K. Buiting et al., "De novo deletions of SNRPN exon 1 in early human and mouse embryos result in a paternal to maternal imprint switch," Nature Genetics, vol. 25, no. 1, pp. 74-78, 2000.

[33] M. Okano, D. W. Bell, D. A. Haber, and E. Li, "DNA methyltransferases Dnmt3a and Dnmt3b are essential for de novo methylation and mammalian development," Cell, vol. 99, no. 3, pp. 247-257, 1999.

[34] K. Hata, M. Okano, H. Lei, and E. Li, "Dnmt3L cooperates with the Dnmt3 family of de novo DNA methyltransferases to establish maternal imprints in mice," Development, vol. 129, no. 8, pp. 1983-1993, 2002.

[35] D. Bourc'his, G.-L. Xu, C.-S. Lin, B. Bollman, and T. H. Bestor, "Dnmt3L and the establishment of maternal genomic imprints," Science, vol. 294, no. 5551, pp. 2536-2539, 2001.

[36] M. Kaneda, M. Okano, K. Hata et al., "Essential role for de novo DNA methyltransferase Dnmt3a in paternal and maternal imprinting," Nature, vol. 429, no. 6994, pp. 900-903, 2004.

[37] T. Arima, K. Hata, S. Tanaka et al., "Loss of the maternal imprint in Dnmt3Lmat-/- mice leads to a differentiation defect in the extraembryonic tissue," Developmental Biology, vol. 297, no. 2, pp. 361-373, 2006.

[38] H. Shizuya, B. Birren, U.-J. Kim et al., "Cloning and stable maintenance of 300-kilobase-pair fragments of human DNA in Escherichia coli using an F-factor-based vector," Proceedings of the National Academy of Sciences of the United States of America, vol. 89, no. 18, pp. 8794-8797, 1992.

[39] X. W. Yang and S. Gong, "An overview on the generation of BAC transgenic mice for neuroscience research," Current Protocols in Meuroscience, vol. 5, chapter 5: unit 5.20, 2005.

[40] S. Swaminathan, H. M. Ellis, L. S. Waters et al., "Rapid engineering of bacterial artificial chromosomes using oligonucleotides," Genesis, vol. 29, no. 1, pp. 14-21, 2001.

[41] S. Warming, N. Costantino, D. L. Court, N. A. Jenkins, and N. G. Copeland, "Simple and highly efficient BAC recombineering using galK selection," Nucleic Acids Research, vol. 33, no. 4, article no. e36, 2005.

[42] S. Gong, X. W. Yang, C. Li, and N. Heintz, "Highly efficient modification of bacterial artificial chromosomes (BACs) using novel shuttle vectors containing the R6K $\gamma$ origin of replication," Genome Research, vol. 12, no. 12, pp. 1992-1998, 2002.

[43] M. Lalioti and J. Heath, "A new method for generating point mutations in bacterial artificial chromosomes by homologous recombination in Escherichia coli," Nucleic Acids Research, vol. 29, no. 3, article no. E14, 2001.

[44] X. W. Yang, P. Model, and N. Heintz, "Homologous recombination based modification in Esherichia coli and germline transmission in transgenic mice of a bacterial artificial chromsome," Nature Biotechnology, vol. 15, no. 9, pp. 859-865, 1997.

[45] L. H. Reid, C. Davies, P. R. Cooper et al., "A 1-Mb physical map and PAC contig of the imprinted domain in $11 \mathrm{p} 15.5$ that contains TAPA1 and the BWSCR1/WT2 region," Genomics, vol. 43, no. 3, pp. 366-375, 1997.

[46] M. Paulsen, K. R. Davies, L. M. Bowden et al., "Syntenic organization of the mouse distal chromosome 7 imprinting cluster and the Beckwith-Wiedemann syndrome region in chromosome 11p15.5," Human Molecular Genetics, vol. 7, no. 7, pp. 1149-1159, 1998.

[47] T. D. Gould and K. Pfeifer, "Imprinting of mouse Kvlqt1 is developmentally regulated," Human Molecular Genetics, vol. 7, no. 3, pp. 483-487, 1998.

[48] R. Kato, H. Shirohzu, T. Yokomine, S.-I. Mizuno, T. Mukai, and H. Sasaki, "Sequence-ready 1-Mb YAC, BAC and cosmid contigs covering the distal imprinted region of mouse chromosome 7," DNA Research, vol. 6, no. 6, pp. 401-405, 1999.

[49] S. Engemann, M. Strödicke, M. Paulsen et al., "Sequence and functional comparison in the Beckwith-Wiedemann region: implications for a novel imprinting centre and extended imprinting," Human Molecular Genetics, vol. 9, no. 18, pp. 2691-2706, 2000.

[50] P. Onyango, W. Miller, J. Lehoczky et al., "Sequence and comparative analysis of the mouse 1-megabase region orthologous to the human 11p15 imprinted domain," Genome Research, vol. 10, no. 11, pp. 1697-1710, 2000.

[51] J. M. Gabriel, T. A. Gray, L. Stubbs, S. Saitoh, T. Ohta, and R. D. Nicholls, "Structure and function correlations at the imprinted mouse Snrpn locus," Mammalian Genome, vol. 9, no. 10, pp. 788-793, 1998.

[52] J. Kim, V. N. Noskov, X. Lu et al., "Discovery of a novel, paternally expressed ubiquitin-specific processing protease gene through comparative analysis of an imprinted region of mouse chromosome 7 and human chromosome 19q13.4," Genome Research, vol. 10, no. 8, pp. 1138-1147, 2000.

[53] S. Hayashida, K. Yamasaki, Y. Asada, E. Soeda, N. Niikawa, and T. Kishino, "Construction of a physical and transcript map flanking the imprinted MEST/PEG1 region at 7q32," Genomics, vol. 66, no. 2, pp. 221-225, 2000. 
[54] J. V. Schmidt, P. G. Matteson, B. K. Jones, X.-J. Guan, and S. M. Tilghman, "The Dlk1 and Gtl2 genes are linked and reciprocally imprinted," Genes and Development, vol. 14, no. 16, pp. 1997-2002, 2000.

[55] S. Takada, M. Tevendale, J. Baker et al., "Delta-like and Gtl2 are reciprocally expressed differentially methylated linked imprinted genes on mouse chromosome 12," Current Biology, vol. 10, no. 18, pp. 1135-1138, 2000.

[56] C. Coombes, P. Arnaud, E. Gordon et al., "Epigenetic properties and identification of an imprint mark in the Nesp-Gnasxl domain of the mouse Gnas imprinted locus," Molecular and Cellular Biology, vol. 23, no. 16, pp. 5475-5488, 2003.

[57] R. M. John and M. A. Surani, "Genomic imprinting, mammalian evolution, and the mystery of egg-laying mammals," Cell, vol. 101, no. 6, pp. 585-588, 2000.

[58] M. Paulsen, T. Khare, C. Burgard, S. Tierling, and J. Walter, "Evolution of the Beckwith-Wiedemann syndrome region in vertebrates," Genome Research, vol. 15, no. 1, pp. 146-153, 2005.

[59] T. Yokomine, H. Shirohzu, W. Purbowasito et al., "Structural and functional analysis of a $0.5-\mathrm{Mb}$ chicken region orthologous to the imprinted mammalian Ascl2/Mash2-Igf2-H19 region," Genome Research, vol. 15, no. 1, pp. 154-165, 2005.

[60] C. A. Edwards, W. Rens, O. Clarke et al., "The evolution of imprinting: chromosomal mapping of orthologues of mammalian imprinted domains in monotreme and marsupial mammals," BMC Evolutionary Biology, vol. 7, article no. 157, 2007.

[61] S. Suzuki, R. Ono, T. Narita et al., "Retrotransposon silencing by DNA methylation can drive mammalian genomic imprinting," PLoS Genetics, vol. 3, no. 4, article no. e55, 2007.

[62] K. Pfeifer, P. A. Leighton, and S. M. Tilghman, "The structural $\mathrm{H} 19$ gene is required for transgene imprinting," Proceedings of the National Academy of Sciences of the United States of America, vol. 93, no. 24, pp. 13876-13883, 1996.

[63] J. F.-X. Ainscough, T. Koidet, M. Tada, S. Barton, and M. A. Surani, "Imprinting of Igf2 and $H 19$ from a $130 \mathrm{~kb}$ YAC transgene," Development, vol. 124, no. 18, pp. 3621-3632, 1997.

[64] A. Wutz, O. W. Smrzka, N. Schweifer, K. Schellander, E. F. Wagner, and D. P. Barlow, "Imprinted expression of the Igf2r gene depends on an intronic CpG island," Nature, vol. 389, no. 6652, pp. 745-749, 1997.

[65] R. M. John, J. F-X. Ainscough, and S. C. Barton, A Transgenic Approach to Studying Imprinted Genes: Modified BACs and PACs, Humana Press, Totowa, NJ, USA, 2002.

[66] M. Ohtsuka, M. Kimura, M. Tanaka, and H. Inoko, "Recombinant DNA technologies for construction of precisely designed transgene constucts," Current Pharmaceutical Biotechnology, vol. 10, no. 2, pp. 244-251, 2009.

[67] F. Cerrato, A. Sparago, I. Di Matteo et al., "The two-domain hypothesis in Beckwith-Wiedemann syndrome: autonomous imprinting of the telomeric domain of the distal chromosome 7 cluster," Human Molecular Genetics, vol. 14, no. 4, pp. 503511, 2005.

[68] R. M. John, M. Hodges, P. Little, S. C. Barton, and M. A. Surani, "A human p57(KIP2) transgene is not activated by passage through the maternal mouse germline," Human Molecular Genetics, vol. 8, no. 12, pp. 2211-2219, 1999.

[69] C. R. Kaffer, A. Grinberg, and K. Pfeifer, "Regulatory mechanisms at the mouse Igf2/H19 locus," Molecular and Cellular Biology, vol. 21, no. 23, pp. 8189-8196, 2001.

[70] C. Gebert, D. Kunkel, A. Grinberg, and K. Pfeifer, "H19 imprinting control region methylation requires an imprinted environment only in the male germ line," Molecular and Cellular Biology, vol. 30, no. 5, pp. 1108-1115, 2010.

[71] R. Schulz, R. B. Mccole, K. Woodfine et al., "Transcriptand tissue-specific imprinting of a tumour suppressor gene," Human Molecular Genetics, vol. 18, no. 1, pp. 118-127, 2009.

[72] D. T. Sowpati, D. Thiagarajan, S. Sharma et al., "An intronic DNA sequence within the mouse Neuronatin gene exhibits biochemical characteristics of an ICR and acts as a transcriptional activator in Drosophila," Mechanisms of Development, vol. 125, no. 11-12, pp. 963-973, 2008.

[73] Y. Obata and T. Kono, "Maternal primary imprinting is established at a specific time for each gene throughout oocyte growth," Journal of Biological Chemistry, vol. 277, no. 7, pp. 5285-5289, 2002.

[74] D. Lucifero, C. Mertineit, H. J. Clarke, T. H. Bestor, and J. M. Trasler, "Methylation dynamics of imprinted genes in mouse germ cells," Genomics, vol. 79, no. 4, pp. 530-538, 2002.

[75] S. Gong, C. Zheng, M. L. Doughty et al., "A gene expression atlas of the central nervous system based on bacterial artificial chromosomes," Nature, vol. 425, no. 6961, pp. 917-925, 2003.

[76] R. M. John, "Engineering mouse models to investigate the function of imprinting," Briefings in Functional Genomics and Proteomics, vol. 9, no. 4, pp. 294-303, 2010.

[77] D. Frank, W. Fortino, L. Clark et al., "Placental overgrowth in mice lacking the imprinted gene lpl," Proceedings of the National Academy of Sciences of the United States of America, vol. 99, no. 11, pp. 7490-7495, 2002.

[78] Y. S. Moon, C. M. Smas, K. Lee et al., "Mice lacking paternally expressed Pref-1/Dlk1 display growth retardation and accelerated adiposity," Molecular and Cellular Biology, vol. 22, no. 15, pp. 5585-5592, 2002.

[79] J. D. Heaney, A. N. Rettew, and S. K. Bronson, "Tissue-specific expression of a BAC transgene targeted to the Hprt locus in mouse embryonic stem cells," Genomics, vol. 83, no. 6, pp. 1072-1082, 2004.

[80] P. Soriano, "Generalized lacZ expression with the ROSA26 Cre reporter strain," Nature Genetics, vol. 21, no. 1, pp. 70-71, 1999. 

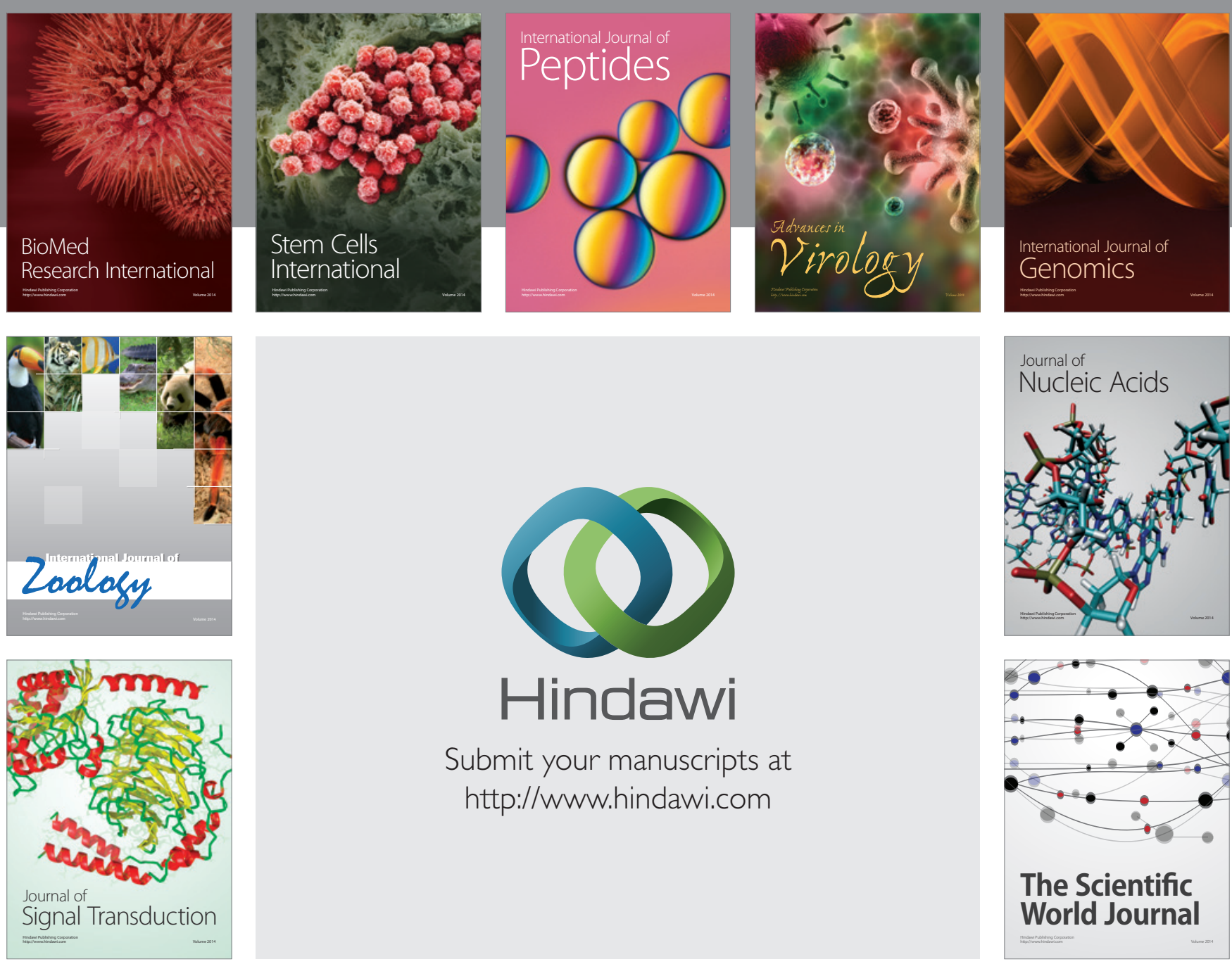

Submit your manuscripts at

http://www.hindawi.com
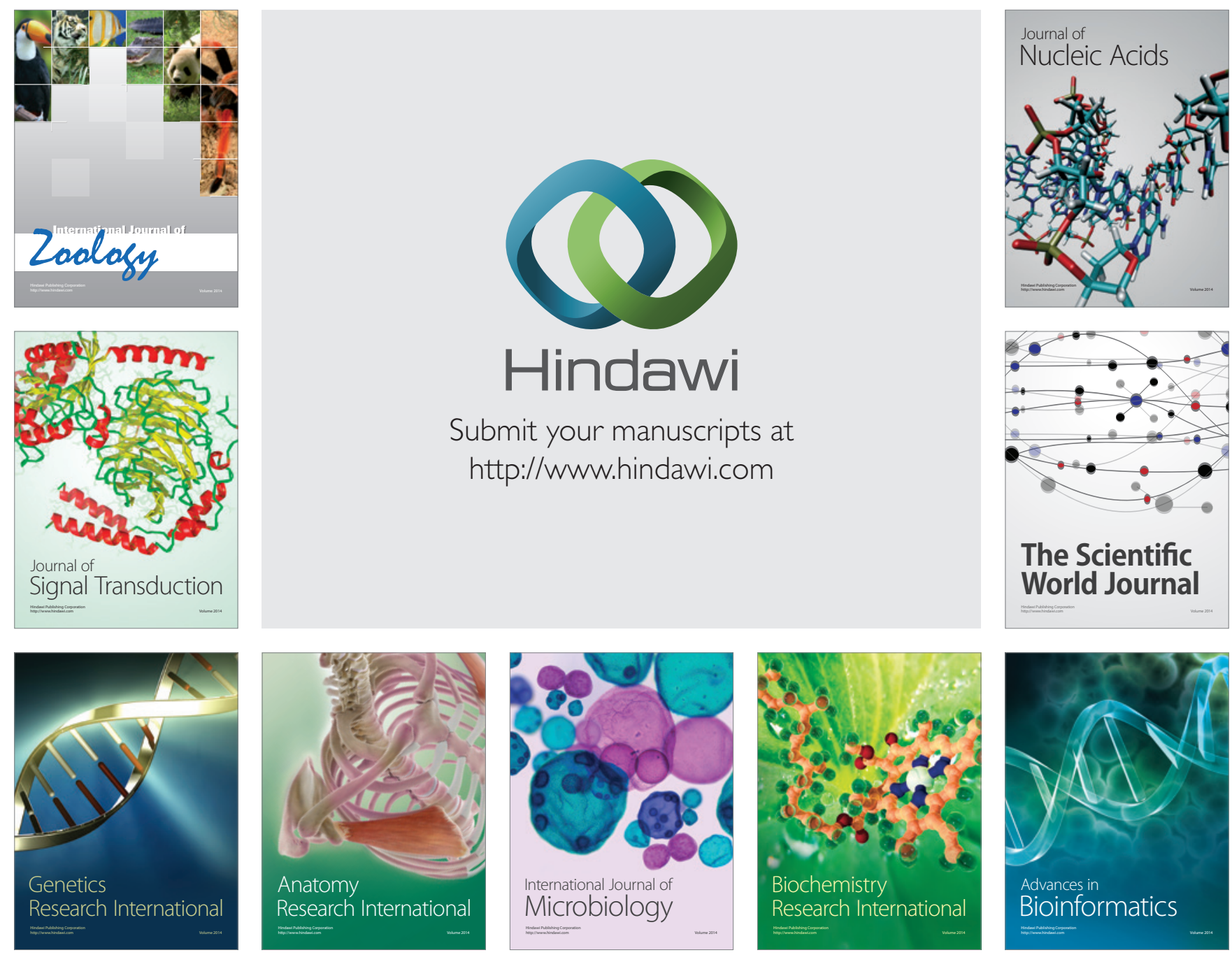

The Scientific World Journal
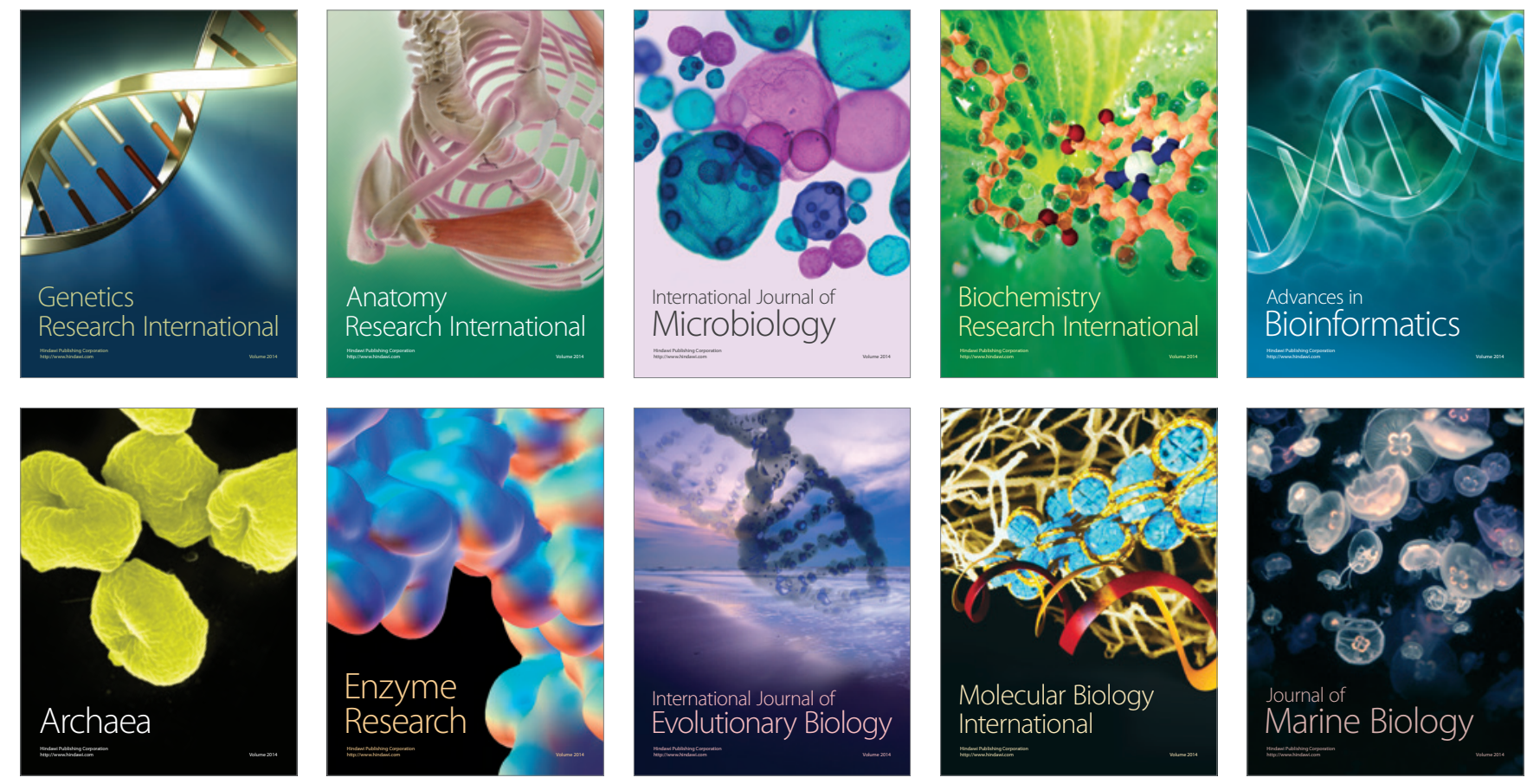